\title{
Papel do cuidador domiciliar na assistência a pessoa com doença de Parkinson: uma revisão integrativa
}

Role of home caregivers in care for persons with Parkinson's disease: an integrative review Papel de los cuidadores domiciliarios en el cuidado de personas con enfermedad de Parkinson: una revisión integradora

Allan Bruno Alves de Sousa Santos ORCID: https://orcid.org/0000-0001-6412-7164

Faculdade de Educação São Francisco, Brasil E-mail: abass@faesf.com.br

Luíza Alves da Silva

ORCID: https://orcid.org/0000-0002-4497-841X Universidade Federal do Piauí, Brasil E-mail: luizaalves.silva83@gmail.com

Maria Eduarda Barros Pardelhas ORCID: https://orcid.org/0000-0001-6881-1959 Universidade Federal do Estado do Rio de Janeiro, Brasil

E-mail: mepardelhas@edu.unirio.br Mikaelle Alves Silva

ORCID: https://orcid.org/0000-0002-9336-1491 Universidade Federal do Espírito Santo, Brasil

E-mail: farm.mikaelle@gmail.com

Sara Paulino dos Anjos

ORCID: https://orcid.org/0000-0002-9568-8666 Universidade Paulista, Brasil

E-mail: sarapaulinodosanjos@hotmail.com

Emanuel Roger dos Santos Reis ORCID: https://orcid.org/0000-0001-7119-4103 Centro Universitário Estácio do Recife, Brasil

E-mail: emanuelrsr@hotmail.com

Ludmila de Araújo Costa

ORCID: https://orcid.org/0000-0002-0810-4424 Faculdade inspirar, Brasil

E-mail: Ludmilaaraujo16@gmail.com

Karine Souza Andrade

ORCID: https://orcid.org/0000-0003-1410-0208

Universidade Cruzeiro do Sul, Brasil

E-mail: karinesouzandrade@icloud.com

Edilson Josué de Oliveira Junior

ORCID: https://orcid.org/0000-0001-5853-6666 Faculdade Guararapes, Brasil

E-mail: oliveiraedilsonjunior@gmail.com

Mariel Wágner Holanda Lima

ORCID: https://orcid.org/0000-0001-5063-3891 Universidade do Estado do Rio Grande do Norte, Brasil E-mail: marielhoolanda@gmail.com

Isabelly Raiane Silva dos Santos

ORCID: https://orcid.org/0000-0002-4034-6677

Universidade Federal do Pará, Brasil

E-mail: isabelly.santos@ifpa.edu.br

Kaline Oliveira de Sousa

ORCID: https://orcid.org/0000-0002-7193-4033

Universidade Federal de Campina Grande, Brasil E-mail: kaline.academico@gmail.com

Julia Suelen Matias dos Santos ORCID: https://orcid.org/0000-0001-8939-1017

Faculdade de Integração do Sertão, Brasil E-mail: juliamatias4@gmail.com

Carlianne Brito dos Santos

ORCID: https://orcid.org/0000-0003-1255-8952

Faculdade Maurício de Nassau Redenção, Brasil

E-mail: carliannebrito@ hotmail.com 
Letícia da Fonseca Anacleto Moreira

ORCID: https://orcid.org/0000-0002-6288-1953

Universidade Federal Fluminense, Brasil

E-mail: leticiaanacleto@id.uff.br

Mateus Silva Soares

ORCID: https://orcid.org/0000-0002-5562-4908

Faculdade de Educação São Francisco, Brasil

E-mail: mateussilva8945@gmail.com

Nair Arrais Leite

ORCID: https://orcid.org/0000-0003-2773-9350

Faculdade de Educação São Francisco, Brasil

E-mail: nairarrais@outlook.com

Leandro Barbosa Teixeira

ORCID: https://orcid.org/0000-0002-9444-7828

Universidade Estácio de Sá, Brasil

E-mail: Lbtteixeira@ hotmail.com

Elias Paulo da Silva

ORCID: https://orcid.org/0000-0001-7583-7567

Universidade Federal do Piauí, Brasil

E-mail: eliaspaulo.silva@gmail.com

Caroline Milanez Paixão

ORCID: https://orcid.org/0000-0002-9669-3109 Universidade Federal do Piauí, Brasil

E-mail: cmilanez18@gmail.com

Islaila Maria Silva Ferreira

ORCID: https://orcid.org/0000-0002-5736-883X Universidade Federal do Piauí, Brasil E-mail: islailamsf28@gmail.com

Bárbara Maria Rodrigues dos Santos

ORCID: https://orcid.org/0000-0002-7335-2408

Universidade Federal do Piauí, Brasil

E-mail: barbara.mariarss@gmail.com

Maria Clara do Nascimento Oliveira

ORCID: https://orcid.org/0000-0002-3860-776X Universidade Federal de Sergipe, Brasil E-mail: clarasgas@gmail.com

Amanda Joyce Alves Silva

ORCID: https://orcid.org/0000-0002-4496-8107

Centro Universitário UNINOVAFAPI, Brasil

E-mail: amandajoyce.alves@gmail.com

Rebeca Rayane de Sousa Marinho

ORCID: https://orcid.org/0000-0002-5566-4889

Faculdade Integrada Brasil Amazônia, Brasil E-mail: rebecarayane225@gmail.com

Jéssica Cristina Lins Zandim

ORCID: https://orcid.org/0000-0002-5408-9800

Universidade Estácio de Sá, Brasil

E-mail: Jhessykhrystynna@gmail.com

Matheus Augusto da Silva Belidio Louzada

ORCID: https://orcid.org/0000-0001-9411-230X

Instituto Fernandes Figueira, Brasil Fundação Oswaldo Cruz, Brasil

E-mail: matheus.belidiolouzada@gmail.com

Thays Almeida da Silva

ORCID: https://orcid.org/0000-0003-2741-3377 Centro Universitário Maurício de Nassau, Brasil E-mail: thays.almeiva@hotmail.com

Elaine Cristina dos Santos Figueira

ORCID: https://orcid.org/0000-0002-4177-9474

Universidade Anhanguera Taubaté, Brasil

E-mail: elaine.figueira88@hotmail.com

Lina Eduarda Silva Costa

ORCID: https://orcid.org/0000-0002-9992-4881

Faculdade Carajás, Brasil

E-mail: linaeduardacosta@gmail.com

Máyra Geovanna Gonzaga Nascimento

ORCID: https://orcid.org/0000-0003-0326-7352

Centro Universitário Tiradentes, Brasil

E-mail: mgeovana_gonzaga@hotmail.com 


\begin{abstract}
Resumo
Buscar na literatura as atividades realizadas pelo cuidador familiar a pessoa com a doença de Parkinson, apontando as suas principais dificuldades e atividades implementadas no domicilio para melhorar a qualidade de vida do paciente. Trata-se de uma revisão integrativa da literatura, de aspecto descritivo e qualitativo. A busca de dados ocorreu nas bases: Literatura Latino-Americana e do Caribe em Ciências da Saúde (LILACS); Scientific Electronic Library Online (SciELO) e Base de Dados de Enfermagem (BDENF). A pesquisa ocorreu no período de junho a julho de 2021, e foram usados os descritores "Doença de Parkinson; Cuidadores; Assistência domiciliar". Estabelecidos na lista dos Descritores em Ciências da Saúde (DECs), e após aplicação dos critérios de elegibilidade restaram oito estudos para serem usados na pesquisa. A principal dificuldade na assistência domiciliar ao paciente com DP é a falta de informações sobre a patologia e por consequência a falta de preparo dos cuidadores, que em sua maioria é composta por cônjuges do sexo feminino. Conclui-se que é necessário haver uma promoção de saúde efetiva no contexto domiciliar, para ofertar qualificação e preparo a esses cuidadores informais.
\end{abstract}

Palavras-chave: Doença de Parkinson; Cuidadores; Assistência domiciliar.

\begin{abstract}
Search the literature for activities performed by family caregivers to people with Parkinson's disease, pointing out their main difficulties and activities implemented at home to improve the patient's quality of life. This is an integrative literature review, with a descriptive and qualitative aspect. The search for data took place in the following databases: Latin American and Caribbean Literature on Health Sciences (LILACS); Scientific Electronic Library Online (SciELO) and Nursing Database (BDENF). The research took place from June to July 2021, and the descriptors "Parkinson's Disease; Caregivers; Home care". Established in the list of Health Sciences Descriptors (DECs), and after applying the eligibility criteria, eight studies remained to be used in the study. The main difficulty in home care for patients with PD is the lack of information about the pathology and, consequently, the lack of preparation of caregivers, who are mostly composed of female spouses. It is concluded that it is necessary to have an effective health promotion in the home context, to offer qualification and preparation to these informal caregivers.
\end{abstract}

Keywords: Parkinson's disease; Caregivers; Home care.

\title{
Resumen
}

Buscar en la literatura las actividades que realizan los cuidadores familiares a las personas con enfermedad de Parkinson, señalando sus principales dificultades y las actividades realizadas en el hogar para mejorar la calidad de vida del paciente. Se trata de una revisión de la literatura integradora, con un aspecto descriptivo y cualitativo. La búsqueda de datos se realizó en las siguientes bases de datos: Literatura Latinoamericana y del Caribe en Ciencias de la Salud (LILACS); Biblioteca Electrónica Científica en Línea (SciELO) y Base de Datos de Enfermería (BDENF). La investigación se llevó a cabo de junio a julio de 2021, y los descriptores "Enfermedad de Parkinson; Cuidadores; Cuidados en el hogar". Establecidos en la lista de Descriptores de Ciencias de la Salud (DEC), y después de aplicar los criterios de elegibilidad, quedaron ocho estudios para ser utilizados en el estudio. La principal dificultad en la atención domiciliaria de los pacientes con EP es la falta de información sobre la patología y, en consecuencia, la falta de preparación de los cuidadores, que en su mayoría están compuestos por mujeres esposas. Se concluye que es necesario contar con una promoción de la salud efectiva en el contexto del hogar, para ofrecer capacitación y preparación a estos cuidadores informales.

Palabras clave: Enfermedad de Parkinson; Cuidadores; Cuidados en el hogar.

\section{Introdução}

A doença de Parkinson (DP), é conceituada como uma enfermidade de caráter neurodegenerativo e progressivo, causada pela diminuição na produção de dopamina, na qual é a responsável pela troca de impulsos entre as células no sistema nervoso, ajudando assim na realização dos movimentos voluntários do organismo, e quando a falta desse neurotransmissor no encéfalo, em uma região específica denominada substância negra, o indivíduo perde o controle motor (Massano, 2011).

O processo de senescência é inevitável para o organismo, no qual percorrem uma série de mudanças, sendo fisiológicas, psicológicas e sociais, na qual todos os indivíduos saudáveis apresentam morte progressiva das células nervosas, e não se sabem os motivos definitivos que favorecem a morte dessas células nervosas e ocasiona o surgimento da patologia de Parkinson, porém acredita-se que seja uma causa multifatorial, contendo fatores genéticos e ambientais, sendo as principais sintomatologias apresentadas são: bradicinesia, rigidez articular, tremores, e também pode surgir as sintomatologias caracterizadas por não serem motoras, tais como alterações do sono, problemas intestinais como a constipação persistente e padrões olfatórios diminuídos (Cabreira \& Massano, 2019). 
Segundo a Organização Mundial da Saúde (OMS), 1\% da população mundial acima dos 65 anos possuem a patologia de Parkinson, com pico na sétima década de vida, sendo mais prevalente no sexo masculino, a uma medida de três homens acometidos, para cada duas mulheres com DP, na região Europeia, estima-se que há de 257 a 1.400 casos por 100 mil habitantes (Vitória, 2021).

No panorama nacional, estima-se que a quantidade de pessoas acometidas pela DP corresponde a 3\% da população acima dos 60 anos, e levando em consideração a quantidade populacional, aponta-se um provável quantitativo de 630 mil brasileiros com DP, e segundo a OMS, o valor pode duplicar até o ano de 2030 (Vitória, 2021).

A DP por se tratar de uma patologia neurodegenerativa, desencadeia ao paciente uma falta de autonomia para desempenhar algumas atividades diárias, necessitando assim de um cuidador em tempo integral, sendo na maioria dos casos, constituído por um membro da família, ou seja, aquele familiar que está mais próximo ao doente, compartilha suas experiências negativas e positivas, e atribui ao paciente uma melhor qualidade de vida no contexto domiciliar (Horning et al, 2019).

Este estudo tem o objetivo de buscar na literatura as atividades realizadas pelo cuidador familiar a pessoa com a doença de Parkinson, apontando as suas principais dificuldades e atividades implementadas no domicilio para melhorar a qualidade de vida do paciente.

\section{Metodologia}

Trata-se de uma revisão integrativa da literatura, de aspecto descritivo e qualitativo. Esse tipo de revisão é utilizado para sintetização de resultados obtidos através de uma determinada pesquisa nas bases de dados selecionadas pelo autor, sobre uma temática específica e se caracterizando por ser sistemática, e sendo usada principalmente em estudos da saúde (Ercole, Melo \& Alcoforado, 2014).

Foi seguida as seguintes etapas para construção do estudo: Elaboração da temática e questão norteadora; seleção dos critérios de inclusão e exclusão; definição dos descritores; definição e busca nas bases de dados; leitura na integra dos manuscritos encontrados, e aplicação dos critérios de elegibilidade; interpretação e agrupamentos dos estudos selecionados.

Para direcionar o estudo, foi estabelecido a seguinte pergunta norteadora " $\mathrm{O}$ que a literatura aborda sobre as experiências dos cuidadores familiares de pacientes com a doença de Parkinson?”.

Na elaboração da pesquisa a busca de artigos ocorreu nas bases de dados Literatura Latino-Americana e do Caribe em Ciências da Saúde (LILACS); Scientific Electronic Library Online (SciELO) e Base de Dados de Enfermagem (BDENF). A pesquisa ocorreu no período de junho a julho de 2021 e foram usados os descritores "Doença de Parkinson; Cuidadores; Assistência domiciliar", estabelecidos na lista dos Descritores em Ciências da Saúde (DECs), e cruzados com o operador booleano “AND”. A estratégia para elaboração do tema e questão norteadora foi a PIco, identificando a população, intervenção e o contexto do estudo.

Foram selecionados os critérios para inclusão de manuscritos para o estudo, sendo utilizado artigos completos disponíveis, com recorte temporal de 2014 a 2021, em idioma português e inglês e que respondessem à questão norte do estudo. E os critérios de exclusão do estudo: artigos duplicados, resumos, teses e dissertações, com recorte temporal inferior a 2014 e que não abordassem o objetivo da pesquisa.

\section{Resultados e Discussão}

No fluxograma a seguir apresenta toda a estratégia de busca, detalhando as principais etapas para a construção da pesquisa, desde as fases iniciais até o resultado final. 
Figura 1. Fluxograma com a trajetória da seleção dos manuscritos.

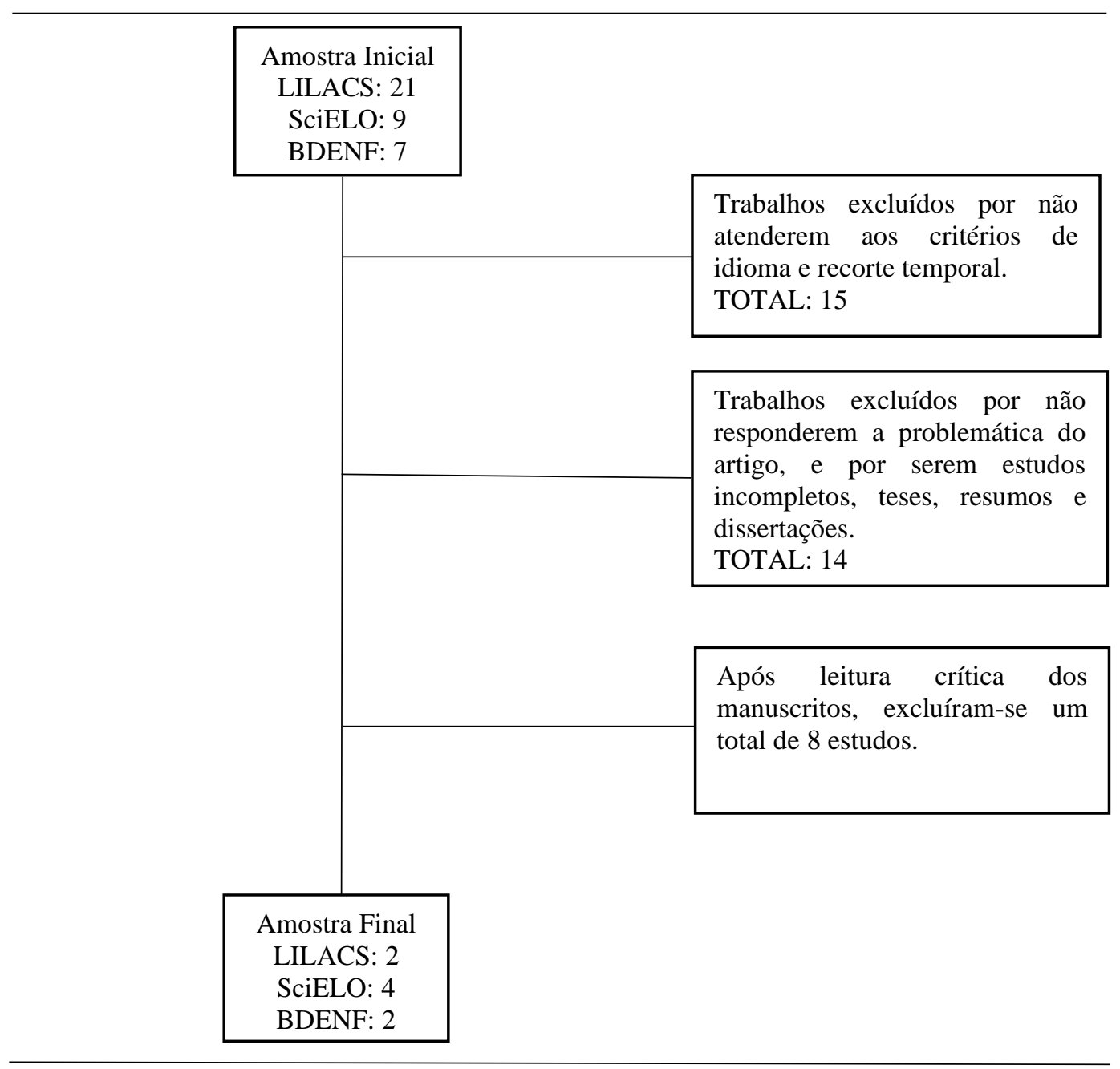

Fonte: Autores (2021).

No Fluxograma 1, observa-se que na busca de dados, constatou-se 37 pesquisas, que foram submetidos a primeira etapa de avaliação por meio dos critérios de elegibilidade. Assim, após aplicar os critérios de recorte temporal e idioma, foram excluídos o quantitativo de 14 estudos. Os estudos foram submetidos a leitura, e foram excluídos 14 estudos por não retratar a temática da pesquisa.

Na segunda etapa, feito a leitura dos manuscritos, observou-se um quantitativo de 8 estudos que respondiam à questão norteadora da pesquisa.

O Quadro 1, a seguir, apresenta os estudos selecionados para a pesquisa segundo sua autoria, título, método, ano e objetivo. 
Quadro 1 - Artigos referentes ao estudo.

\begin{tabular}{|c|c|c|c|c|}
\hline Autor & Título & Método & Ano & Objetivo \\
\hline $\begin{array}{l}\text { Nunes, } \\
\text { Alvarez, Costa } \\
\text { \& Valcarenghi. }\end{array}$ & $\begin{array}{lll}\text { Adaptação } & \text { dos } & \text { familiares } \\
\text { Cuidadores } & \text { de Idosos com } \\
\text { Doença } & \text { de } & \text { Parkinson: } \\
\text { Processo de Transição. }\end{array}$ & $\begin{array}{l}\text { Método exploratório de } \\
\text { abordagem qualitativa. }\end{array}$ & 2019 & $\begin{array}{l}\text { Descrever e interpretar a repercussão da } \\
\text { transição para o exercício do papel de cuidador } \\
\text { em familiares de idosos com doença de } \\
\text { Parkinson, à luz da teoria das Transições de } \\
\text { Afaf Meleis. }\end{array}$ \\
\hline $\begin{array}{l}\text { Nunes, } \\
\text { Alvarez, Costa } \\
\text { \& Valcarenghi. }\end{array}$ & $\begin{array}{lrr}\text { Fatores } & \text { determinantes } & \text { na } \\
\text { transição } & \text { situacional } & \text { de } \\
\text { familiares } & \text { cuidadores } & \text { de } \\
\text { idosos com doença } & \text { de } \\
\text { Parkinson. }\end{array}$ & $\begin{array}{l}\text { Estudo qualitativo, } \\
\text { exploratório e descritivo. }\end{array}$ & 2019 & $\begin{array}{l}\text { Investigar os fatores facilitadores e inibidores } \\
\text { na transição situacional de familiares cuidadores } \\
\text { de idosos com doença de Parkinson. }\end{array}$ \\
\hline Padovani et al. & $\begin{array}{l}\text { Ser cuidador de pessoas com a } \\
\text { Doença de Parkinson: } \\
\text { situações vivenciadas }\end{array}$ & $\begin{array}{l}\text { Estudo qualitativo } \\
\text { análise temática. }\end{array}$ & 2018 & $\begin{array}{l}\text { Compreender a experiencia de cuidar de uma } \\
\text { pessoa com a doença de Parkinson. }\end{array}$ \\
\hline Gondim et al. & $\begin{array}{l}\text { Conhecimentos, atitudes e } \\
\text { práticas de pessoas com } \\
\text { doença de Parkinson sobre um } \\
\text { programaraperutico } \\
\text { domiciliar com orientação em } \\
\text { grupo. }\end{array}$ & $\begin{array}{l}\text { Estudo de corte transversal } \\
\text { e abordagem quantitativa. }\end{array}$ & 2018 & $\begin{array}{l}\text { Investigar os conhecimentos, as atitudes e as } \\
\text { práticas de pessoas com a DP sobre um } \\
\text { programa de exercícios terapêticos } \\
\text { domiciliares autos supervisionados com } \\
\text { orientação em grupo. }\end{array}$ \\
\hline $\begin{array}{l}\text { Alvarez, } \\
\text { Valcarenghi, } \\
\text { Nunes, Siewert } \\
\text { \& Baptista. }\end{array}$ & $\begin{array}{l}\text { O impacto da aposentadoria } \\
\text { nas pessoas com doença de } \\
\text { Parkinson em idade ativa. }\end{array}$ & Pesquisa qualitativa. & 2017 & $\begin{array}{l}\text { Compreender o impacto da aposentadoria nas } \\
\text { pessoas com doença de Parkinson em idade } \\
\text { ativa. }\end{array}$ \\
\hline $\begin{array}{l}\text { Maia, Galvão, } \\
\text { Oliveira \& \& } \\
\text { Miranda. }\end{array}$ & $\begin{array}{l}\text { Assistência à pessoa com } \\
\text { Parkinson no âmbito da } \\
\text { estratégia de saúde da família. }\end{array}$ & $\begin{array}{l}\text { Estudo descritivo- } \\
\text { exploratória, com } \\
\text { abordagem qualitativa. }\end{array}$ & 2016 & $\begin{array}{l}\text { Analisar as concepções que o portador da } \\
\text { Doença de Parkinson (DP) detém sobre a } \\
\text { assistência integral realizada pelo enfermeiro. }\end{array}$ \\
\hline Alvarez et al. & $\begin{array}{l}\text { Grupo de apoio às pessoas } \\
\text { com doença de Parkinson e } \\
\text { seus familiares. }\end{array}$ & Relato de experiência & 2016 & $\begin{array}{l}\text { Descrever a experiência do projeto de extensão } \\
\text { - Grupo de apoio às pessoas com doença de } \\
\text { Parkinson e seus familiares no âmbito da UFSC. }\end{array}$ \\
\hline $\begin{array}{l}\text { Filippin, } \\
\text { Martins, Dela } \\
\text { Libera, } \\
\text { Halberstadt \& } \\
\text { Severo. }\end{array}$ & $\begin{array}{l}\text { Qualidade de vida de sujeitos } \\
\text { com doença de Parkinson e } \\
\text { seus cuidadores. }\end{array}$ & Estudo transversal. & 2014 & 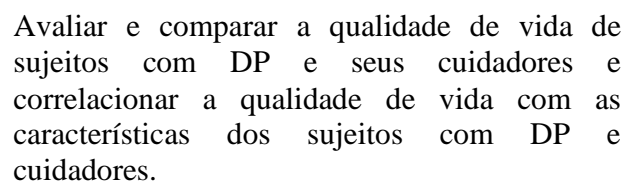 \\
\hline
\end{tabular}

Fonte: Autores (2021).

A evolução da doença de Parkinson provoca sintomas que afeta toda a constituição familiar, alterando sua rotina e suas prioridades, na qual se destaca que o cuidador exerce todas as tarefas relacionadas ao cuidado diário do paciente, desempenhando seu papel de cuidador domiciliar em tempo integral, não apresenta temor pela DP, pois não tem conhecimentos sobre a sintomatologia e progressão da patologia (Padovani et al, 2018).

O sentimento vivenciado pelos cuidadores não é algo estático, pois varia em intensidade conforme a progressão da doença, tais como solidão, medo, culpa e tristeza, na qual como consequência também aumenta o grau de dependência do paciente, e mesmo com a sobrecarga de emoções, em estudos feitos por Padovani et al (2018), os cuidadores priorizam o próprio cuidado, realizando atividades como caminhadas, natação e realizam exames laboratoriais de rotina.

Segundo os estudos de Nunes et al (2017), a descoberta do diagnóstico de DP, causa um impacto na vida dos familiares, isso devido aos sintomas da patologia, dificultar em alto grau a capacidade do paciente em desempenhar atividades de vida diária, sendo o cônjuge o principal cuidador. Em conformidade com os estudos de Padovani et al (2018), os cuidadores são em sua maioria constituído pelo cônjuge, esse fato acontece principalmente pelo grau de convívio e parentesco.

A uma carência de informação aos cuidadores sobre o entendimento da doença, e seus principais agravos, o que dificulta o cuidado domiciliar, com a educação em saúde, o cuidador pode planejar sua assistência, realizar técnicas de relaxamento e combate ao stress, otimizar o tempo no desenvolvimento de auxílio as tarefas diárias e principalmente realizar 
um preparo para lidar com a progressão da doença no futuro, a assim consolidando a importância e necessidade de se trabalhar a educação desses cuidadores no contexto domiciliar (Nunes et al, 2017). Enfatizado pelos estudos de Godin et al (2018), na qual aponta a importância de se incentivar o autocuidado, e explicar ao paciente com DP, a necessidade de se ter uma vida ativa, com práticas de exercício diários, objetivando retardar a evolução degenerativa da doença de Parkinson.

A espiritualidade e religiosidade encoraja os cuidadores e proporciona esperança, na qual é justificado pelos estudos de Nunes et al (2017), quando se apontar que ter uma crença em algo, ajuda o cuidador a enfrentar as situações estressoras e dificuldades da assistência diária, enfatizando que o apoio de amigos e vizinhos também se caracteriza fator importante no cuidado ao paciente com DP.

A quantidade de tempo em horas gastos pelo cuidador, na assistência ao paciente com DP, supera as horas gastas com o próprio cuidado, significando que o cuidador precisa fazer vários reajustes a sua vida pessoal para conseguir promover uma assistência domiciliar íntegra e qualitativa, o que pode ocasionar danos a sua saúde mental, e assim torna-se necessário e aconselhável a indicação de grupos de apoio ao cuidador domiciliar (Alvarez et al, 2016)

Os aspectos sociais, emocionais e físicos, são os principais fatores desencadeantes de uma piora na qualidade de vida, e em especial vivenciados com intensidade no período de transição, de familiar para cuidador (Filippin et al, 2014). E assim os estudos de Nunes et al (2017), enfatiza a dificuldade que os familiares encontram em passar pela transição e se tornarem um cuidador domiciliar, na qual inicia-se com a conscientização das primeiras manifestações da patologia, e a adaptação a uma nova rotina diária.

A visita domiciliar feita pela equipe multiprofissional de saúde, é fator importante para a oferta de qualidade de vida ao paciente, proporcionando uma educação em saúde a família e atendimento holístico, sanado assim todas as eventuais incertezas do paciente e do cuidador (Galvão, Oliveira, Maia \& Miranda, 2016). Analisando os estudos de Alvarez et al (2017), a assistência especializada em saúde no contexto domiciliar, é importante à medida que proporciona ao paciente um cuidado holístico e ocorre a disponibilidade de todas as assistências ofertadas pelo Sistema Único de Saúde (SUS), desde as consultas na Unidade Básica de Saúde até a beneficência com questões de aposentadorias, assuntos que não pertencem as atividades domiciliares dos cuidadores familiares.

Como limitação deste estudo, pode-se realçar a poucas quantidades de estudos que abordassem com clareza as experiencias vivenciadas pelos cuidadores no contexto domiciliar as pessoas que convivem com a Doença de Parkinson, e a importância de se educar essa população de cuidadores sobre como desempenhar com êxito as práticas essenciais do cotidiano.

\section{Considerações Finais}

Este estudo é importante à medida que proporciona compreender as atividades desenvolvidas no âmbito domiciliar, para melhorar a qualidade de vida da pessoa com doença de Parkinson. Com o conhecimento adquirido através da revisão integrativa, observa-se que o grupo de cuidadores domiciliares é em sua maioria constituídos por cônjuges do sexo feminino, na qual a principal dificuldade encontrada é a falta de preparo e desinformação sobre a doença.

Ressalta-se a necessidade de haver uma promoção de saúde efetiva no contexto domiciliar, para ofertar qualificação e preparo a esses cuidadores informais, nesse contexto o estudo alcançou excelência em apontar as experencias vivenciadas pelos cuidadores no âmbito familiar.

Este estudo é importante à medida que contribui para novas produções sobre a temática, e que possa favorecer futuramente para uma comunidade de cuidadores domiciliares mais informada e qualificada. 


\section{Referências}

Acta Medica Portuguesa. SUP2011, 24, 827-834.

Acta Medica Portuguesa. Out2019, 32, 661-670.

Alvarez, A. M., Valcarenghi, R. V., Nunes, S. F. L., Siewert, J. S., \& Baptista, R. (2017). O impacto da aposentadoria nas pessoas com doença de Parkinson em idade ativa. Revista Eletrônica De Enfermagem, 19. https://doi.org/10.5216/ree.v19.39607

Alvarez, A., Gonçalves, L., Schier, J., Hammerschmidt, K., Souza, B., \& Valcarenghi, R. (2016). Grupo de apoio às pessoas com Doença de Parkinson e seus familiares. Extensio: Revista Eletrônica De Extensão, 13(22), 92. 10.5007/1807-0221.2016v13n22p92

Dia Mundial de Conscientização da Doença de Parkinson | Biblioteca Virtual em Saúde MS. (n.d.). Biblioteca Virtual em Saúde MS. https://bvsms.saude.gov.br/dia-mundial-de-conscientizacao-da-doenca-de-parkinson/

Dia Mundial de Combate ao Mal de Parkinson: como perceber sinais antecipadamente - Hospital Santa Mônica. (2020). Hospital Santa Mônica. https://hospitalsantamonica.com.br/prioridade-dia-mundial-de-combate-ao-mal-de-parkinson/

Ercole, F., Melo, L., \& Alcoforado, C. (2014). Integrative review versus systematic review. Reme: Revista Mineira De Enfermagem, 18(1). 10.5935/14152762.20140001 .

Filippin, N., Martins, J., Dela Libera, L., Halberstadt, B., \& Severo, A. (2014). Qualidade de vida de sujeitos com doença de Parkinson e seus cuidadores. Fisioterapia Em Movimento, 27(1), 57-66. 10.1590/0103-5150.027.001.ao06

Gondim, I., Lins, C., Asano, N., Curi, D., Lins, O., \& Coriolano, M. (2018). Conhecimentos, Atitudes E Práticas De Pessoas Com Doença De Parkinson Sobre Um Programa Terapeûtico Domicilar Com Orientação Em Grupo. Estudos Interdisciplinares Sobre O Envelhecimento, 23(2). 10.22456/2316-2171.76898

Gonçalves, L. H. T., Alvarez, A. M., \& Arruda, M. C. (2007). Pacientes portadores da doença de Parkinson: significado de suas vivências. Acta Paulista de Enfermagem, 20(1), 62-68. https://doi.org/10.1590/s0103-21002007000100011

Maia, C., Galvão, T., Oliveira, K., \& Miranda, F. (2021). Assistência à pessoa com Parkinson no âmbito da estratégia de saúde da família Assistance to the person with Parkinson carrier in the context of family health strategy.

Nunes, S., Alvarez, A., Costa, M., \& Valcarenghi, R. (2019). Determining Factors In The Situational Transition Of Family Members Who Care Of Elderly People With Parkinson's Disease. Texto \& Contexto - Enfermagem, 28. 10.1590/1980-265x-tce-2017-0438

Nunes, S., Alvarez, A., Valcarenghi, R., Hammerschmidt, K., \& Baptista, R. (2019). Adaptação dos Familiares Cuidadores de Idosos com Doença de Parkinson: Processo de Transição. Psicologia: Teoria E Pesquisa, 35(spe). 10.1590/0102.3772e35nspe4

O que é a doença de Parkinson? (artigo) | Khan Academy. (n.d.). Khan Academy. https://pt.khanacademy.org/science/health-and-medicine/nervous-systemdiseases/parkinsons-disease/a/what-is-parkinsons-disease

Padovani, C., Lopes, M., Higahashi, I., Pelloso, S., Paiano, M., \& Christophoro, R. (2018). Being caregiver of people with Parkinson's Disease: experienced situations. Revista Brasileira De Enfermagem, 71(suppl 6), 2628-2634. 10.1590/0034-7167-2017-0008. https://www.einstein.br/doencas-sintomas/parkinson

Vitória, R. (2021). Dia Mundial do Parkinson: segunda doença neurodegenerativa mais prevalente no mundo. https://www.folhavitoria.com.br/saude/noticia/04/2019/dia-mundial-do-parkinson-segunda-doenca-neurodegenerativa-mais-prevalente-no-mundo

Horning, M. A., Shin, J. Y., DiFusco, L. A., Norton, M., \& Habermann, B. (2019). Symptom progression in advanced Parkinson's disease: Dyadic perspectives. Applied nursing research, ANR, 50, 151193. https://doi.org/10.1016/j.apnr.2019.151193 